\title{
Geodetic survey as a means of improving fast MASW (Multichannel Analysis Of Surface Waves) profiling in difficult terrain/land conditions
}

\author{
Rafal Matuła ${ }^{1, *}$, Paulina Lewińska ${ }^{2}$ \\ ${ }^{1}$ AGH University of Science and Technology, Faculty of Drilling, Oil and Gas, Krakow, Poland \\ ${ }^{2}$ AGH University of Science and Technology, Faculty of Mining Surveying and Environmental Engineering, Krakow, Poland
}

\begin{abstract}
This paper revolves around newly designed and constructed system that can make 2D seismic measurement in natural, subsoil conditions and role of land survey in obtaining accurate results and linking them to 3D surface maps. A new type of land streamer, designed for shallow subsurface exploration is described in this paper. In land seismic data acquisition methods a vehicle tows a line of seismic cable, lying on construction called streamer. The measurements of points and shots are taken while the line is stationary, arbitrary placed on seismic profile. Exposed land streamer consists of 24 innovatory gimballed $10 \mathrm{~Hz}$ geophones. It eliminates the need for hand 'planting' of geophones, reducing time and costs. With the use of current survey techniques all data obtained with this instrument are being transferred in to 2D and 3D maps.

This process is becoming more automatic.
\end{abstract}

\section{Introduction}

Since the CMP-style (Common Middle Point) methodology was introduced into engineering seismic profiling, improving the efficiency of seismic shallow data acquisition was discussed. It has been an important factor for most seismic production and research projects. Improvements can be discussed in regard to: automation, mobility, consistency and safety of seismic sources. Advancements in engineering technologies facilitated all of those factors to some extent. Over years, shallow seismic acquisition methods, and for the most part acquisition equipment, have been reduced in size and shape so to resemble prospecting equipment and methods. This has mainly been a necessity for the economy of small scale projects. Recent developments in shallow seismic imaging efficiency have taken advantage of highresolution, surveying methods. This includes automated systems which incorporate sources and receivers designed for land applications with towed techniques, adopted from marine seismic acquisition. With combining modern, engineering acquisition systems' components and an awareness of the shallow seismic technology's potential, the acquisition costs (measured by required time) could be reduced up to 50 percent. Several experimental land acquisition systems have been developed to atomise following processes: roll and unroll seismic cable, plant and retrieve conventional spiked geophones into ground, haul fixed spreads of receivers along the ground surface maintaining source and receiver offset without invasive, manually positioned geophones. Researchers for over two decades attempted to find a way to tow long receiver spreads across the ground surface in a way similar to marine sea-streamers. This would allow to transfer efficiency of seismic surveying to land [2], [3], [8] Shear wave land streamers were also developed and tested for near-surface applications during the late 1990s [9]. Land streamers, that towed arrays or spreads, have primarily been designed and tested for applications of so called continuous seismic profiling. Low signal-to-noise ratios and the need for achieving highest frequency signals possible, are characteristic to shallow seismic surveys. Their volume has limited the widespread use in many near-surface settings of land streamer technologies. It is worth to mention that towed spread designs are suited for the high signal-to-noise ratio and lower frequencies.

This paper describes a towed spread developed for multichannel analysis of surface waves (MASW) [1]. The spread is designed to be hauled by the source vehicle and pressure coupled to various surfaces (varying from asphalt and lightly vegetated to loose soils). Comparisons of data acquired with the towed spread and conventional, coupled geophones is also given in this article. Appraise between the trade-off conventional "planting" of geophones for surface wave registration is described.

Nowadays more emphasis is being put on combing data from differed sources in to one 2D or 3D model. Specialists from various technical fields see the need of interdisciplinary works that would create one, complete picture of surveyed objects and structures with regard to there special placement. This is why geodetic/survey personnel is often being welcomed into data acquisition groups - since it can provide a space for correlation and comparison of various datasets. Described new data acquisition method is no different at this point. In order to

*Corresponding author: rmatula@agh.edu.pl 
connect the subsurface analysis performed by geophysics to pre-existing topographic maps GPS [4], [5] survey was required. It had to be done within time frame, and conditions facilitated by this new and fast geophysics method. What is more, all gathered information had to be implemented and stored in to one special data file thus allowing for fast access to all data. GPS receivers allow fast acquisition of spatial data curtail for correctly placing obtained geological profiles.

\section{Device description}

The prototype of described land streamer system is built with parts intended for application in various, conditions and acquisition techniques. Self-orienting, gimbalmounted geophones with $10 \mathrm{~Hz}$ sensors on half spherical underlays were recording the seismic wave arrivals. The receivers were mounted in $\sim 2.5 \mathrm{~kg}$ underlays to ensure geophone-to-ground coupling. The mentioned geophones with underlays were connected by belts made from synthetic material, resistant to stretching. Signals were transferred to seismograph by cable that had takeout's for 48 geophones at $2 \mathrm{~m}$ intervals. Cables are terminated with robust waterproof connectors. The towed land streamer was limited by the 48-channel capacity of these connectors. The projected streamer construction provides possibility of adding an extra weight in case a need of increasing geophone-to ground coupling appears. Performed experiments have demonstrated that geophone-to-geophone coupling via the half-sphere underlays is possible and very effective for the porpoise of surveying open, natural areas. To take full advantage of the efficiency of the towed land streamer acquisition system, a fast, self-propelled sources that provide strong signal (characterized by high and low frequencies) was required [19].

For surveys described in this article a sledge-hammer based, Seismic Source Company source was used. Mentioned seismic source was mounted on tracked tractor (IHI35), which was used for towing land-streamer. Used acquisition system was configured for imaging relatively shallow and intermediate depth targets, simultaneously. Half-automatic scanning of the subsurface using surface waves can be done cost effectively and with confidence at relatively high production rates if: consistent time-lapse measurement can be made, high production rates can be maintained, and fixed processing flows can be used on all data sets from a particular area.

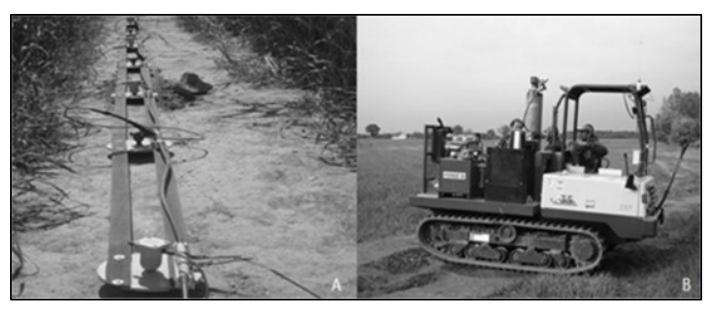

Fig. 1. In section A - part of projected land streamer, B seismic source used for surveys.

Acquisition test have proven that coupling necessary for accurate recording of surface waves can be established and maintained over an extended distance and variable terrain with just pressure contact to the earth's surface through aggressive contact points. Unlike standard surveying, where coupling of geophones by direct "planting" produces the processed data, surface wave energy recorded from planted geophones is equivalent (for most applications) to surface wave energy recorded from pressure coupled geophones.

\section{Brief description of device application procedure}

Acquisition schema with the towed land streamer is similar to technique on which the marine surveys was primarily based $[3,4,6,9]$. In contrast to the continuous movement of marine survey systems, the operational procedure of the towed land streamer is based on a "excitation technique at a standstill" tactic with some projected acquisition step [17, 7, 8, 14, 15]. In Figure 1A, the streamer comprises of one module, with forty eight 2 $\mathrm{m}$ takeout's is shown. Initially in standard survey methodology case study, layout of receivers is kept at a fixed location, and shot positions are moved from back to front of the seismic spread (Fig. 2B) [5, 10]. In comparison to land streamer, once the shot position reaches the front, it is kept at a fixed distance related to the first receiver's position. Sources and receivers are shifted simultaneously after each shot (Fig. 2A) [1, 2]. The streamer is moved up one shot interval, the shot is detonated once the streamer has been stationary for a few seconds, and the process repeated up to the end of profile. Since moving the land streamer generates significant noise, including variable vertical accelerations, there is no possibility to shoot while it is in motion. Comparable acquisition procedures using spike-mounted geophones and standard common-midpoint (CMP) cables are more labour intensive $[10,13,18]$. To record with 48 active channels (as for the exposed land-streamer example), a minimum of two 24 takeout engineering cables is required. At least one 48- channel standard cable is necessary for conventional procedures using a roll-along device to change active channels [16].

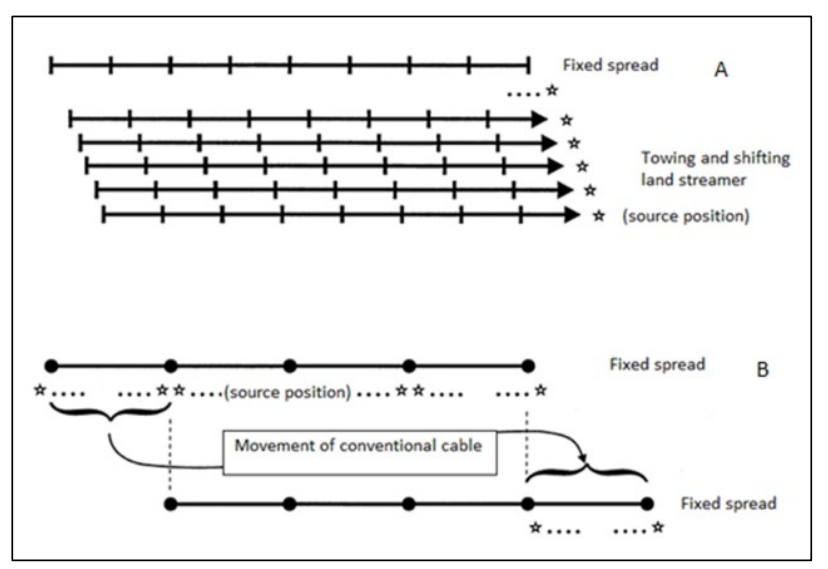

Fig. 2. Land streamer measurement realization (A), CMP-style surveying (B). 
One profile was undergoing detailed examination. The survey geometry was modified for obtaining very highresolution data. $2 \mathrm{~m}$ receiver spacing was used for every acquisition schema. Each shot-points were separate in every 12 meters for roll-along technique, with seismic spread movement equal to $1 / 4$ spread length (schematic example in Fig. 2B). Land streamer survey interval was at 5 meter between each position of projected acquisition system. Measurements were made for surveys with the sledgehammer-type source described in the section above.

In general, line preparation and deployment and the retrieval of geophones and cables are most time consuming aspects of conventional strategy of seismic surveys. For an efficient conventional shallow seismic survey on land, a field crew of five people is needed: one person operating the recording equipment, two managing the seismic source, two geophone/cable layers. For a comparable land-streamer survey, only three members of field personnel are required (as during the traditional survey) but as operator doubles since the driver of the towing vehicle and the geophone/cable layers are not required. Compared to the traditional approach, the towed land-streamer system was about $50-90 \%$ faster and required only about $30-40 \%$ need of personnel. In other words, the land streamer surveys on the same profile were completed with $25-50 \%$ of the person-hours required for the traditional surveys. During described test about 200 records were obtained during four hour land streamer measurements. Using the half-automatic sledge hammer source, acquisition speed of the towed land-streamer seems to be faster ( $\sim 2$ times without repeating) than the vibrator technique.

\section{Comparison of towed spread geophones and conventional spike coupled geophones}

Evaluating how effective a towed spread records seismic data is relative to conventionally planted geophones can be done through analysis of comparable data. Ideally these data are recorded using the same source energy and as nearly consistent ground surface conditions as possible. For this study, two records - part of CMP-style record and landstreamer- made record, were selected with equal receiver positions and uniquely near-surface conditions and characteristics of part of seismic profile. Both data were simultaneously recorded using both spike planted geophones and pressure coupled streamer geophones. For the Polish lowland site both receiver configurations were coupled to as near identical soil as possible. However, under natural condition conventional spread was planted above the road track while the towed spread was drug along road's surface. Underground structure of chosen site has relatively small differences, and can be used as test site for planted and streamer geophones. It is almost completely without near-surface static inconsistency between adjacent receiver locations. Surface wave analysis usually involves calculation of dispersion. It is the first and most efficient diagnostic step in the processing flow of surface wave data [3]. Subtle wavelet differences are evident in waveforms and arrival consistency when comparing equivalent shot gathers from planted geophones and geophones in the streamer. Due to the reduced trace-to-trace consistency in waveforms on the streamer data, the amplitudes appear to vary more than spectral analysis suggests from dispersion energy analysis [3]. Seismic data designed and acquired for surface wave analysis were recorded using the same seismic source energy for both the towed array and conventional spikeplanted geophone spreads.
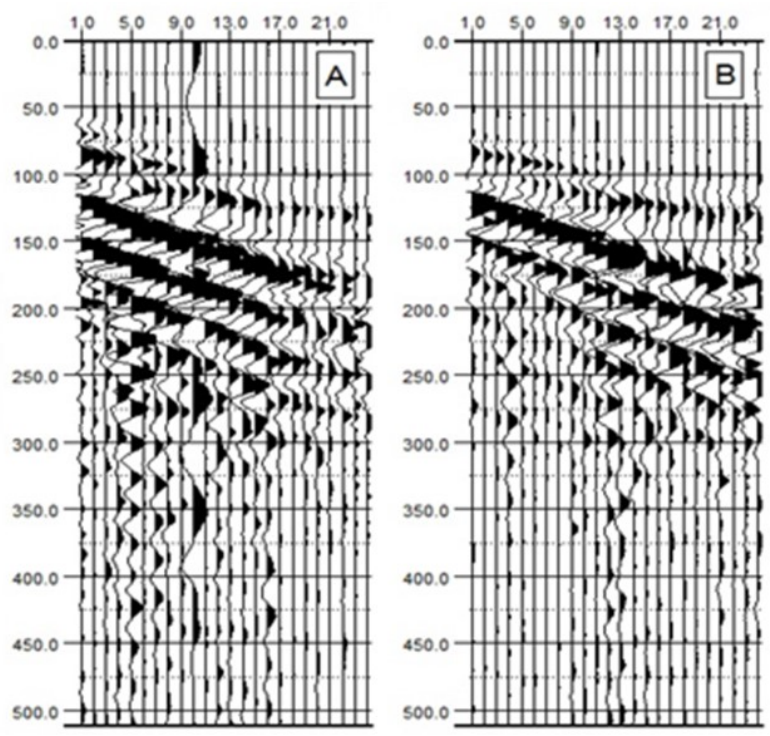

Fig. 3. Examples parts (half of all active channels of central part of records) of obtained records. In section A data recorded by using land streamer. In section $\mathrm{B}$, normal surveying with spiked into ground geophones. On the vertical axis time in [msec].

Geophones recording surface waves on the road surface using the towed array have a slightly lower dominant frequency, higher ratio of surface waves to body waves, and a much more coherent and fully developed dispersive surface wave. It is unlikely the coupling is improved with the land streamer, but recording on the road surface does improve the quality (trace-to-trace consistency and amplitude) and quantity of surface wave energy captured. Dispersion energy analysis reinforces this observation with a much larger amplitude and better defined fundamental mode than with the land streamer data. An apparent break in slope of the surface wave arrival near the middle of the spread appears to manifest itself on dispersion energy analysis as the break in slope observed at about $15 \mathrm{~Hz}$. Increased noise at higher frequencies on conventional geophones is evident in dispersion energy analysis. Clearly the road represents much more uniform media than the variably sculpted, eroded, and infilled road rut, therefore producing a dispersion curve with minimal interference from trace-totrace shift. 


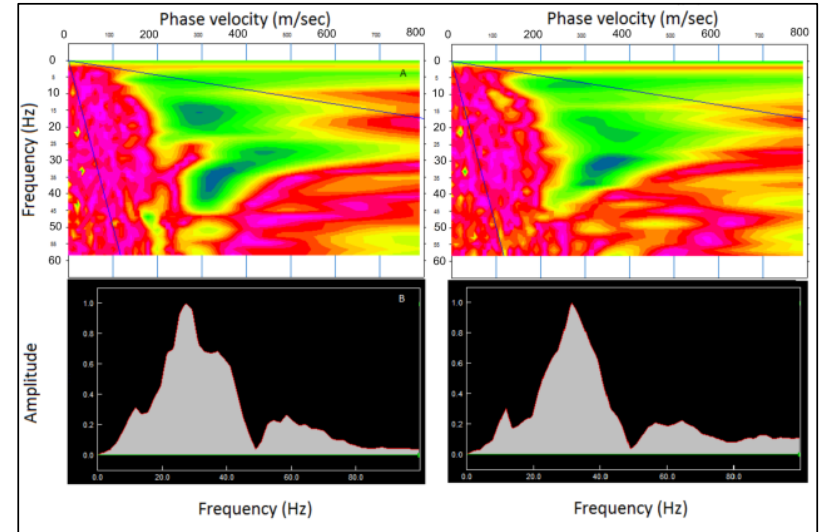

Fig. 4. A and B spectrum and dispersion curve of spiked geophones data. $\mathrm{C}$ and $\mathrm{D}$ the distribution for land streamer data.

During presented tests a RTK-GPS survey of measured lines was performed. The technique employed in both methods was the same however during MASW survey it needed to be much faster and done almost on the go. GPS survey consisted of placing 3 GPS reference stations that would cover the area of geophysics works. This allowed the crew to be independent from Polish GPS corrections sourced during the actual surveys. It was checked beforehand that the area was placed unfavourably in regard to them. The main task was to cared out GPS-RTK surveys during the movement of geophone-units every few meters in order to establish position of the line and selected units.

Also it was important not to get in the way with geophysics crew. GPS data was downloaded on the side and implemented in to further processing's. All gathered data, survey and geophysics, ware connected in to one 3D model and stored as one data set. Also georeferenced 2D maps an cross-sections ware created. This allows for better visualisation on how subsoil structure influences the surface and constructions placed upon it.

\section{Conclusions}

In situations where Multichannel Surface Wave Analysis (MASW) is used as a main methodology for image of subsurface material, a towed array on the paved surface can produce better data than spike-planted geophones in natural terrain conditions. For surface wave analysis where the ground surface is conducive to spike-planted geophones, a towed spread produces equivalent data quality. All aspects considered, this towed spread design is very effective in recording good consistent surface wave energy in most surface settings. With the source as the tow vehicle and the seismograph attached to the rear of the spread, data can be acquired rapidly and surface wave processing can be accomplished using fixed flows in almost real time. Body wave analysis will in many surface settings be possible, providing good preliminary images and maps of the surface and adding GPS coordinates will allow for better 3D placement of acquired data. What is more coordinates can be downloaded on side and be implemented during post-processing and visualisation of geophysics data thus allowing other specialist for better interpretation of the results.

The project was funded by statutory funds of AGH no. 11.11.150.005 and 11.11.190.555.

\section{References}

1. A.J. Kruppenbach, J.W. Bedenbender, Towed land cable: U.S. Patent 3923121 (1975); Towed land cable: U.S. Patent 3954154 (1976)

2. A.J.M. Pugin, T.H. Larson, S.L. Sargent, J.H. McBride, C. E. Bexfield, The Leading Edge 23 (7), 677 (2004)

3. C.B. Park, J. Xia, R.D. Miller, 68th Ann. Internat. Mtg. Soc. Expl. Geophys., Expanded abstracts, 1377 (1998)

4. D. Einarsson, L. Brooks, G. Bennett, A. White, Geophysics 42, 148 (1977) - abstract

5. D.W. Steeples, Mining Engineering, 1297 (October 1993)

6. I. Gausland, The Leading Edge 19 (8), 903 (2000)

7. J. Ivanov, R.D. Miller, P. Lacombe, C.D. Johnson, Geophysics 71, A39 (2006)

8. J.M. Lorenzo Saanumi, C. Westbrook, S. Egnew, 76th Ann. Internat. Mtg. Soc. Expl. Geophys., Expanded abstracts, 1496 (2006)

9. J. Caldwell, W. Dragoset, The Leading Edge 19 (8), 898 (2000)

10. K.H. Waters, Reflection seismology - A tool for energy resource exploration (New York, 1987)

11. K. Maciuk, Geodetski vestnik 60 (3), 455 (2016)

12. K. Maciuk, Artificial Satellites 51 (1), 1 (2016)

13. M.A. Speece, S.J. Betterly, R.H. Levy, 76th Ann. Internat. Mtg. Soc. Expl. Geophys., Expanded abstracts, 1 (2007)

14. M. Van der Veen, A.G. Green, Geophysics 63, 1408 (1998)

15. M. Van der Veen, Buness, H.A. Buker, A.G. Green, J. Env. Eng. Geophys. 5, 39 (2000)

16. T. Ghunaim Al-Anezi, M. AlMalki, T. Alkhalifa, Arabian Journal of Geosciences 6 (1), 235 (2013)

17. T. Inazaki, Ann. Symp. Environ. Engin. Geophys. Soc. (SAGEEP), Expanded abstracts, 207 (1999)

18. T. Inazaki, Near Surface 2006, Expanded abstracts, A016 (2006)

19. Y. Sawasdeen, Geophysical Prospecting 57 (1), 125 (2009) 